\title{
STABILITY AND ATTRACTIVITY OF SOLUTIONS OF DIFFERENTIAL EQUATIONS WITH IMPULSES AT FIXED TIMES ${ }^{1}$
}

\author{
S. SIVASUNDARAM \\ Embry-Riddle Aeronautical University \\ Department of Computing and Mathematics \\ Daytona Beach, FL 32114 USA \\ E-mail: Siva@db.erau.edu \\ S. VASSILYEV \\ Russian Academy of Sciences \\ Institute of System Dynamics and Control Theory \\ Irkutsk 664033, Russia \\ E-mail:vas@icc.ru
}

(Received October, 1997; Revised February, 1999)

In this paper we consider the dynamics of solutions of impulsive differential equations with fixed time moments of impulsive effects on the basis of comparison methods and vector Lyapunov functions. We propose sufficient conditions on the following dynamic properties: stability, attractivity, and some combinations of them.

Key words: Stability, Attractivity, Impulse, Differential Equations, Lyapunov Functions, Comparison Methods.

AMS subject classifications: 34D05, 34A30.

\section{Introduction}

In [1] it has been proposed to study the stability of solutions of impulsive differential equations on the basis of comparison method and Vector Lyapunov Functions (VLF). In this paper, we obtain more weak sufficient conditions of stability and extend this approach onto more wide class of dynamic properties. The main aim of this paper is to show that on the basis of comparison methods which is rather traditional, now it is very easy to obtain sufficient conditions of different dynamic properties. In Section 2 , we describe mathematical models of analyzed system and comparison system. In Section 3, we state and prove Wazewski-like result on differential inequalities. For dynamic properties which definitions are introduced in Section 4, we propose in

\footnotetext{
${ }^{1}$ This work was supported in part by the NRC grant 15043-7199 and RFBR grant 98-01-01137.
}

Printed in the U.S.A. (C2000 by North Atlantic Science Publishing Company 
Section 5, new sufficient conditions in terms of VLF. Some illustrative examples are considered in Section 6 .

\section{Mathematical Models}

Consider an increasing sequence $t_{1}, t_{2}, \ldots$ of reals $t_{k} \in R_{0}^{1}=[0,+\infty), k \in N=1,2, \ldots$, such that $t_{k} \rightarrow+\infty$ when $k \rightarrow+\infty$ and a function $\varphi: R_{0}^{1} \times R^{p} \rightarrow R^{q}$. We denote $\varphi\left(t_{k}^{+}, z\right) \stackrel{\text { def }}{=} \lim _{h \rightarrow 0+} \varphi\left(t_{k}+h, z\right)$ if the right side limit exists. The function $\varphi$ is said to belong to class $\Phi_{p q}$ if

(i) $\varphi$ is continuous in $\left(t_{k-1}, t_{k}\right] \times R^{p}$, and

(ii) $\forall k \in N \forall z \in R^{p} \exists u \in \bar{R}^{q}$

$$
u=\lim _{\substack{\boldsymbol{w} \rightarrow 0+\\ w \rightarrow z}} \varphi\left(t_{k}+h, w\right)
$$

Let us consider two systems of differential equations with impulsive effects [1]

$$
\begin{gathered}
\left\{\begin{array} { c c } 
{ x ^ { \prime } = F ( t , x ) , \quad t = t _ { k } , } & { F \in \Phi _ { n n } } \\
{ x ( t _ { k } ^ { + } ) = x ( t _ { k } ) + I _ { k } ( x ( t _ { k } ) ) , } & { t = t _ { k } } \\
{ x ( t _ { 0 } ^ { + } ) = x _ { 0 } , } & { }
\end{array} \left\{\begin{array}{cc}
y^{\prime}=f(t, y), \quad t \neq t_{k}, & t=\Phi_{m m} \\
y\left(t_{k}^{+}\right)=\psi_{k}\left(y\left(t_{k}\right)\right), &
\end{array} y\left(t_{0}^{+}\right)=y_{0},\right.\right.
\end{gathered}
$$

where $k \in N$, and solutions $x=x\left(\cdot, t_{0}, x_{0}\right), y=y\left(\cdot, t_{0}, y_{0}\right)$ are to be understood as left continuous functions with points of discontinuity of the first kind at $t=t_{k}$. More exactly [1], a function $x:\left(t_{0}, t_{0}+\Delta\right) \rightarrow R^{n}, t_{0} \geq 0, \Delta>0$, is said to be a solution of (2.1) if

(i) $\quad x\left(t_{0}^{+}\right)=x_{0}, \forall t \in\left[t_{0}, t_{0}+\Delta\right)(t, x(t)) \in d o m F$,

(ii) $\quad x$ is continuously differentiable and satisfies the equality

$$
x^{\prime}(t)=F(t, x(t)) \text { for } t \in\left[t_{0}, t_{0}+\Delta\right), \quad t \neq t_{k},
$$

(iii) if $t=t_{k} \in\left[t_{0}, t_{0}+\Delta\right)$, then $x\left(t^{+}\right)=x(t)+I_{k}(x(t))$, and at such $t$ 's, $x(t)$ is left continuous.

For the system (2.2) its solutions $y(t)$ are to be understood analogously and in addition, we assume that the function $f(t, y)$ satisfies in the field of its definition, the Wazewski condition in $y[3]$, i.e., possesses the following property of quasimonotone nondecreasing:

if $\forall(t, x),\left(t, x^{\prime}\right) \in d o m f$ such that $x \leq x^{\prime}, \forall i \in 1, \ldots, m$ such that $x_{i}=x_{i}^{\prime}$

$$
f_{i}(x) \leq f_{i}\left(x^{\prime}\right)
$$


(vector inequalities are to be understood componentwise).

\section{Lemma on Differential Inequality}

The systems (2.1), (2.2) are connected by a function $v(t, x), v \in \Phi_{n m}$ which is locally Lipschitzian in $x$ and satisfies the differential inequality

$$
\bar{D}^{+} v(t, x) \leq f(t, v(t, x)), t \neq t_{k}, k \in N
$$

In addition, we introduce the following requirement:

$$
\begin{gathered}
\forall k \in N \quad \forall x \in X_{k} \quad \forall y \in Y_{k} \\
v\left(t_{k}, x\right) \leq y \Rightarrow v\left(t_{k}^{+}, x+I_{k}(x)\right) \leq \psi_{k}(y)
\end{gathered}
$$

where $X_{k}, Y_{k}$ are the regions of achievability of the systems (2.1), (2.2) respectively at the time moment $t_{k}$. If the sets $X_{k}$ are unknown, then we replace in the (3.2) the set $X_{k}$ by $R^{n}$ (analogously, $Y_{k}$ can be replaced by $R^{m}$ ).

A solution $y\left(\cdot, t_{0}, y_{0}\right)$ of $(2.2)$ is said to be upper solution and is noted by $\bar{y}\left(\cdot, t_{0}, y_{0}\right)$ if for any solution $\tilde{y}\left(\cdot, t_{0}, y_{0}\right)$ of $(2.2)$ with the same initial data $t_{0}, y_{0}$

$$
\forall t \in \operatorname{dom} \bar{y} \cap \operatorname{dom} \tilde{y} \quad \tilde{y}\left(\cdot, t_{0}, y_{0}\right) \leq \bar{y}\left(\cdot, t_{0}, y_{0}\right)
$$

Lemma 3.1: If the systems (2.1), (2.2), and the locally Lipschitzian in $x$ function $v: R_{0}^{1} \times R^{n} \rightarrow R^{m}, \quad v \in \Phi_{n m}$, satisfy the conditions (2.3), (3.1), (3.2), then for all $\left(t_{0}, x_{0}\right) \in \operatorname{dom} F, \quad\left(t_{0}, y_{0}\right) \in \operatorname{domf}$ such that $v\left(t_{0}, x_{0}\right) \leq y_{0}$, there exists an upper solution of $(2.2) \tilde{y}=\bar{y}\left(\cdot, t_{0}, y_{0}\right)$ such that for any solutions $x=x\left(\cdot, t_{0}, x_{0}\right)$ of $(2.1)$

$$
\forall t \in d o m x \cap \operatorname{dom} \bar{y} \quad v(t, x(t)) \leq \bar{y}(t) .
$$

Proof: Let the conditions of Lemma 3.1 be satisfied, $\left(t_{0}, x_{0}\right) \in \operatorname{dom} F,\left(t_{0}, y_{0}\right) \in$ domf, $v\left(t_{0}\right) \leq y_{0}, t_{i}$ be the minimal of time moments $t_{k}, k \in N$, such that $t_{0}<t_{i}$.

Let $t_{0} \notin\left\{t_{k}: k \in N\right\}$. Consider (2.2) (only differential equation) on time interval $\left[t_{0}, t_{i}\right]$. In this case, the existence of the solution and upper solution of the initial value problem $y^{\prime}=f(t, y), y\left(t_{0}\right)=y\left(t_{0}^{+}\right)=y_{0}$ depends only on the function $f$ and we are now in the position to apply Wazewski theorem [3] and to claim that there exists a time interval $\left[t_{0}, \tau\right), \tau \leq t_{i}$, on which the upper solution $\bar{y}\left(\cdot, t_{0}, y_{0}\right)$ exists.

If the $\left[t_{0}, \tau\right)$ is maximal interval of existence of the solution $\bar{y}$ of the system (2.2) on the whole field of its definition, then $\bar{y}$ goes to infinity when $t \rightarrow \tau^{-}$, and by the same theorem, we obtain that Lemma 3.1 is valid on an interval $\left[t_{0}, \tau\right) \cap$ domx.

Let the solutions $\bar{y}, x$ be determined at $t_{i}$ also. Then $\bar{y}\left(t_{i}^{+}\right)$is determined and equal $\psi_{i}\left(\bar{y}\left(t_{i}\right)\right)$. We can consider the condition $\bar{y}\left(t_{i}^{+}\right)=\psi_{i}\left(\bar{y}\left(t_{i}\right)\right)$ as the new initial condition of solutions of the differential equation from (2.2) and by the Wazewski theorem we can prove that there exists a time interval $\left[t_{i}, \tau_{1}\right), \tau_{1} \leq t_{i+1}$ where the upper solution $\bar{y}\left(\cdot, t_{i}, \bar{y}\left(t_{i}^{+}\right)\right)$exists. On the basis of $(3.2)$

$$
v\left(t_{i}, x\left(t_{i}\right)\right) \leq \bar{y}\left(t_{i}\right)
$$




$$
\Rightarrow v\left(t_{i}^{+}, x\left(t_{i}^{+}\right)\right)=v\left(t_{i}^{+}, x\left(t_{i}\right)+I_{i}\left(x\left(t_{i}\right)\right) \leq \psi_{i}\left(\bar{y}\left(t_{i}\right)\right)=\bar{y}\left(t_{i}^{+}\right)\right.
$$

Therefore, for any solution $x\left(\cdot, t_{i}, x\left(t_{i}\right)+I_{i}\left(x\left(t_{i}\right)\right)\right)$ and for all $t \in \operatorname{dom} x \cap\left[t_{i}, \tau_{1}\right)$ using the same theorem [3] we obtain the inequality

$$
v(t, x(t)) \leq \bar{y}(t)
$$

and so on. Repeating this, we finally arrived at the desired inequality for all $t \in$ $d o m x \cap \operatorname{dom} \bar{y}$.

If for some $k \in N, t_{0}=t_{k}$, then the initial value problem $\bar{y}\left(t_{0}^{+}\right)=y_{0}$ for differential equation from (2.2) and majorizing condition (3.3) are considered as above for the corresponding problems on interval $\left[t_{i}, t_{i+1}\right]$ and the proof is complete.

Remark 3.1: If $v\left(t_{k}^{+}, x\right)=v\left(t_{k}, x\right), \forall k \in N$, e.g., $v$ does not depend on $t$ or is right continuous in $t$, and to assume that

(a) the functions $\psi_{k}$ are nondecreasing,

(b) $v\left(t_{k}, x+I_{k}(x)\right)=\psi_{k}\left(v\left(t_{k}, x\right)\right)$

then the condition (3.2) is valid for all $x \in R^{n}, y \in R^{m}$.

\section{Dynamic Properties}

Assume that in the system (2.1), the following dynamic property is studied

$$
\begin{gathered}
\forall t_{0} \in T^{0} \subseteq R_{0}^{1} \quad \exists Q \in \mathscr{R}_{1}^{0} \quad \forall P \in \Re \\
{\left[\left(\exists P^{0} \in \mathscr{R}_{1}^{0} \quad \forall x_{0} \in M\left(P^{0}, Q, t_{0}\right) \quad \forall x=x\left(\cdot, t_{0}, x_{0}\right)\right.\right.} \\
\left.\forall t \geq t_{0}: t \in \operatorname{domx} \quad x(t) \in P(t)\right) \&\left(\forall x_{0}\right) \in Q\left(t_{0}\right) \forall x\left(\cdot, t_{0}, x_{0}\right) \\
\left.\left.\exists t_{1} \geq t_{0}\left(\forall t \geq t_{1}: \quad t \in d o m x\right) \quad x(t) \in P(t)\right)\right]
\end{gathered}
$$

where $\forall P^{0} \in \mathscr{B}_{i}^{0} P^{0} \subseteq T^{0} \times R^{n}, i \in 1,2, \forall P \in \mathscr{B} \quad P \subseteq R_{0}^{1} \times R^{n}$ and $M=M\left(P^{0}, Q, t_{0}\right)$ is some subset of $R^{n}$, dependent on $P^{0}, Q, t_{0}$.

I. Assume particularly that $M\left(P^{0}, Q, t_{0}\right)=P^{0} \backslash Q\left(t_{0}\right)$ and consider the following cases:

(i) When $\mathfrak{R}_{1}^{0}$-single-point set, $Q(t) \equiv Q^{*}$ is fixed, $Q^{*}=R_{0}^{n}=\left\{x \in R^{n}\right.$ : $x \geq 0\}$,

$$
\Re_{0}=\mathscr{R}_{2}^{0}=\mathscr{R}^{*}=\left\{R_{0}^{1} \times P_{\epsilon}: \epsilon>0\right\}, P_{\epsilon}=\left\{x \in R^{n}:\|x\|<\epsilon\right\},
$$

the property (4.1) means stability or attractivity of solutions. More exactly, this means the attractivity to the origin of solutions with nonnegative vectors of initial states and stability of other solutions.

(ii) When $\mathfrak{R}_{2}^{0}=\{\emptyset\}, Q(t)=Q^{*}$ is fixed, then (4.1) is equivalent to $Q^{*} \mathfrak{B}_{-}$ attractivity. 
(iii) When $Q(t) \equiv \emptyset$, the property (4.1) means $\mathscr{B}_{\mathscr{B}_{2}}^{0}$-stability [4].

(iv) If $Q(t) \equiv \emptyset$ and $\mathscr{R}=\{P\}$-singl, $\mathscr{R}_{2}^{0}=\{P\}$-singl, then (4.1) defines the property of practical stability, or more exactly, $P P^{0}$-estimation of solutions [4].

II. If in (4.1), $M\left(P^{0}, Q, t_{0}\right)=P^{0}\left(t_{0}\right)$, then under the assumptions of the case $(i)$, we obtain the definition of the property of asymptotic stability.

\section{Comparison Theorem and Corollaries}

The system (2.1) and function $v \in \Phi_{n m}$ are said to be comparison system and comparison function respectively for the system (2.1) if the majorizing condition (3.3) is valid. In the system (2.2) we consider the property $\left(4.1_{c}\right)$ which has the same meaning and is described in the same fashion in terms of corresponding sets $T_{c}^{0} \subseteq R_{0}^{1}$,

$\mathscr{R}_{c} \subseteq$ $2^{R_{0}^{1} \times R^{m}}, Q_{c} \subseteq \mathscr{R}_{1 c}^{0}, \mathscr{R}_{i c}^{0} \subseteq 2^{T_{c}^{0} \times R^{m}}, \ldots$ We assume further that $T^{0}=T_{c}^{0}$.

Theorem 5.1: Let for the system (2.1), there exist comparison system (2.2) and comparison function $v \in \Phi_{n m}$ such that the following conditions are valid:

(1) $\forall t_{0} \in T^{0} \quad \forall Q_{c} \in \mathscr{R}_{1 c}^{0} \quad \exists Q \in \mathscr{R}_{1}^{0}\left[\forall P_{c}^{0} \in \mathscr{R}_{2 c}^{0} \exists P^{0} \in \mathscr{R}_{2}^{0} \forall x_{0} \in M \quad \exists y_{0} \in M_{c}\right.$ $\left.v\left(t_{0}, x_{0}\right) \leq y_{0} \& \forall \forall x_{0} \in Q\left(t_{0}\right) \exists y_{0} \in Q_{c}\left(t_{0}\right) v\left(t_{0}, x_{0}\right) \leq y_{0}\right] ;$

(2) $\forall t_{0} \in T^{0} \forall y_{0} \in R^{m}$ there exists the upper solution $\bar{y}\left(\cdot, t_{0}, y_{0}\right)$ of (2.2) infinitely right continuable;

(3) $\forall t_{0} T^{0} \quad \forall P \in \Re_{B} \exists P_{c} \in \Re_{c} \forall t \geq t_{0} x \notin P \& y \in P_{c} \Rightarrow v(t, x) \not y$; then $\left(4.1_{c}\right) \Rightarrow(4.1)$.

Corollary 5.1: (the case (4.1.I.i)). Let in (4.1) $M=P^{0} \backslash Q\left(t_{0}\right), Q(t) \equiv Q^{*}$, the set $Q^{*}$ be fixed, $\Re_{0}=\Re_{2}^{0}=\Re^{*}$, the corresponding sets in $\left(4.1_{c}\right)$ be analogously chosen and the following conditions be satisfied for all $t_{0} \in T^{0}$ :

$$
\begin{gathered}
\forall \epsilon>0 \exists \delta>0 v\left(t_{0}, P_{\delta} \backslash Q^{*}\right) \subseteq P_{\epsilon c} \backslash Q_{c}^{*} ; \\
v\left(t_{0}, Q^{*}\right) \subseteq Q_{c}^{*} ; \\
\forall \epsilon>0 \quad \exists \delta>0 \quad \forall t \geq t_{0} \quad \forall x \in R^{n} \backslash P_{\epsilon} \quad \forall y \in P_{\delta c} v(t, x) \not \leq y .
\end{gathered}
$$

Assume also that the conditions of Lemma 3.1 are satisfied and in the sense of (2) upper solutions of (2.2) exist and are infinitely right continuable. (Conditions a). Then $\left(4.1_{c}\right) \Rightarrow(4.1)$, i.e. (2.1) possesses the property of stability or attractivity (see the case, I.i above).

Corollary 5.2: (the case (4.1.II)). Let all the assumptions of Corollary 5.1 be satisfied except that: $M=P^{0}\left(t_{0}\right), Q$ si not fixed (analogously in $\left(4.1_{c}\right)$ ), and instead of (5.1), (5.2) the conditions below are valid:

$$
\begin{gathered}
\forall \epsilon>0 \quad \exists \delta>0 \quad v\left(t_{0}, P_{\delta}\right) \subseteq P_{\epsilon c} \\
\forall Q_{c} \in \mathscr{R}_{1 c}^{0} \quad \exists Q \in \mathscr{R}_{1}^{0} \quad v\left(t_{0}, Q\right) \subseteq Q_{c} .
\end{gathered}
$$

Then $\left(4.1_{c}\right) \Rightarrow(4.1)$, i.e., asymptotic stability of (2.2) implies the analogous property of (2.1). 
Corollary 5.3: (4.1.I.ii). Let in (4.1) $\mathscr{R}_{2}^{0}=\emptyset$ and for all $t_{0} \in T^{0}$ the conditions (5.3), (5.5), (a) be satisfied. Then $\Re_{1 c}^{0} \Re_{c}$-attractivity of (2.2) implies $\Re_{1}^{0} \Re_{\text {-attrac- }}$ tivity of (2.1).

Corollary 5.4: (4.1.I.iii). Let in (4.1) $\mathfrak{B}_{0}=\mathfrak{B}_{2}^{0}=\mathfrak{B}^{*}, Q(t) \equiv \emptyset, M=P^{0}\left(t_{0}\right)$ and for all $t_{0} \in T^{0}$ the conditions (5.3), (5.4) as well as conditions (a) are satisfied. Then stability of (2.2) implies stability of (2.1).

Corollary 5.5: (4.1.I.iv). Let in (4.1) $\mathscr{B}=\{P\}$-singl, $\Re_{0}^{0}=\left\{P^{0}\right\}$-singl, $Q(t) \equiv \emptyset$, $M=P^{0}\left(t_{0}\right)$. Assume that the conditions $(a)$ are satisfied and for all $t_{0} \in T^{0}$ the following conditions are valid:

$$
\begin{gathered}
v\left(t_{0}, P^{0}\left(t_{0}\right)\right) \subseteq P_{c}^{0}\left(t_{0}\right) \\
\forall t \geq t_{0} \quad \forall x \in R^{n} \backslash P(t) \quad \forall y \in P_{c}(t) \quad v(t, x) \not \leq y .
\end{gathered}
$$

Then $P_{c} P_{c}^{0}$-estimation of (2.2) implies $P P^{0}$-estimation of (2.1).

\section{Examples}

Let us consider some illustrative examples which have been analyzed in [2] also.

Example 6.1: For the following model

$$
\left\{\begin{array}{c}
x_{1}^{\prime}=x_{2}, \\
x_{2}^{\prime}=-x_{1}-\gamma x_{1}^{3}, \quad \gamma>0 \text { when } t \neq t_{k} \\
\Delta x_{1}=\alpha_{k} x_{1}, \\
\Delta x_{2}=\beta_{k} x_{2} \quad \text { when } t=t_{k}
\end{array}\right.
$$

where $\Delta x_{i}=x_{i}\left(t_{k}^{+}\right)-x_{i}\left(t_{k}\right), i=1,2$, let us use the function $v\left(x_{1}, x_{2}\right)=\frac{1}{2} x_{1}^{2}+$ $\frac{1}{4} \gamma x_{2}^{4}+\frac{1}{2} x_{2}^{2}$.

Since $\bar{D}^{+} v(x)=0$, we obtain comparison system

$$
\left\{\begin{aligned}
y^{\prime} & =0 \\
y\left(t_{k}^{+}\right) & =y\left(t_{k}\right),
\end{aligned}\right.
$$

where second relationship is derived from majorizing of $v(x+\Delta x)$.

Condition (3.2) is satisfied if

$$
v\left(x_{1}+\alpha_{k} x_{1}, x_{2}+\beta_{k} x_{2}\right) \leq v\left(x_{1}, x_{2}\right)
$$

This can be satisfied by $\alpha_{k} \in[-1,0], \beta_{k} \in[-1,0]$. Trivial solution of the comparison system (6.2) is stable. All other conditions of Corollary 5.4 are satisfied. Therefore, the trivial solution of the considered system is also stable.

In [2], the example like (6.1) has been analyzed not from the viewpoint of stability, but asymptotic stability and instability on the basis of some other theorems, which contain other conditions stated in terms of Lyapunov function (not in terms of comparison function and without comparison system). 
Example 6.2: Consider the model

$$
\left\{\begin{array}{cc}
x_{1}^{\prime}=-2 x_{2}+x_{2} x_{3}-x_{1}, & \\
x_{2}^{\prime}=x_{1}-x_{1} x_{3}-x_{3}, & \text { when } t \neq t_{k} \\
x_{3}^{\prime}=x_{1} x_{2}-x_{3}, & \text { when } t=t_{k} \\
\Delta x_{i}=d_{i} x_{3} &
\end{array}\right.
$$

where $t_{k}=k T, k \in N, T>0, d_{i}>0, i=1,2,3$.

Let us use the function $v(x)=x_{1}^{2}+2 x_{2}^{2}+x_{3}^{2}$. Then we shall obtain $\bar{D}^{+} v(x)=-2 v(x)$ and the comparison system

$$
\left\{\begin{array}{c}
y^{\prime}-2 y \\
y\left(t_{k}^{+}\right)=\psi_{k}\left(y_{k}\right)
\end{array}\right.
$$

where $\psi_{k}$ is subject to find.

Let $d_{\text {max }}=\max \left\{d_{1}, d_{2}, d_{3}\right\}$. Then

$$
\begin{gathered}
v\left(x+I_{k}(x)\right)=\left(1+d_{1}\right)^{2} x_{1}^{2}+2\left(1+d_{2}\right)^{2} x_{2}^{2}+\left(1+d_{3}\right)^{2} x_{3}^{2} \\
\leq\left(1+d_{\text {max }}\right)^{2} x_{1}^{2}+2\left(1+d_{\text {max }}\right)^{2} x_{2}^{2}+\left(1+d_{\text {max }}\right)^{2} x_{2} \\
=\left(1+d_{\text {max }}\right)^{2} v(x) ; \\
\psi_{k}=\left(1+d_{\text {max }}\right)^{2} y_{k} .
\end{gathered}
$$

Variation of $y$ on $T$ as decreasing is equal to $y\left(t_{k-1}\right)\left(1-e^{-2 T}\right)$, therefore $\Delta y=$ $\psi_{k}\left(y_{k}\right)-y\left(t_{k}\right)$ has to be equal or less then $y\left(t_{k-1}\right)\left(1-e^{-2 T}\right)$ if we want to have stability of (6.4). Thus,

$$
\begin{gathered}
\Delta y=y\left(t_{k}\right)\left(2 d_{\text {max }}+d_{\text {max }}^{2}\right)=y\left(t_{k-1}\right) e^{-2 T}\left(2 d_{\max }+d_{\max }^{2}\right) \\
\leq y\left(t_{k-1}\right)\left(1-e^{-2 T}\right)
\end{gathered}
$$

and therefore

$$
d_{\max } \leq e^{T}-1
$$

If this inequality is valid, then the (6.3) is stable. In [2] the example (6.3) has been considered also from different viewpoint only for asymptotic stability and instability.

For asymptotic stability of $\mathrm{f}(6.4)$ and as a consequence of the system (6.3), we require $\left.\Delta y<y\left(t_{k-1}\right) 1-e^{-2 T}\right)$ strictly, i.e., $d_{\max }<e^{T}-1$. The last condition coincides with the same result of [2], but it was obtained by a different method (without comparison system and comparison function). 


\section{Conclusion}

In this paper we have considered the system of impulsive differential equations (2.1) and its dynamical properties generalized by the definition (4.1). Under particular cases of choosing the sets contained in (4.1), it is possible to obtain definitions of such different classical dynamical properties as stability, attractivity, invariance, etc., as well as their various combinations like conjunctive property (4.1.II) of asymptotic stability, disjunctive property (4.1.I.i) stability or attractivity, and so on. We have obtained new sufficient conditions for (4.1), and such its particular cases as stability, attractivity and some other dynamic properties of impulsive differential equations (2.1) where time moments of impulsive effects are fixed. These criteria can be more preferable than known ones [1,2], since in general, they can be easier to satisfy. For example, instead of conditions (3.4), (3.5) from [1] we require only (3.2). It is very east to extend these results on different other dynamic properties. It is more difficult to analyze the model with unfixed moments of impulses, particularly when these moments depend on truth of some logical conditions. This logical-dynamic model will be considered in forth coming papers.

\section{References}

[1] Lakshmikantham, V., Bainov, D.D. and Simeonov, P.S., Theory of Impulsive Differential Equations, World Scientific Publishers, Singapore 1989.

[2] Liu, X., Impulsive stabilization of nonlinear systems, J. of Math. Control and Inform. 10 (1993), 11-19.

[3] Wazewski, T., Systemes des equations et des inequalities differentielles ordinaries aux deuxiemes members motones et leurs applications, Ann. Soc. Pol. Math. 23 (1950), 112-166. 


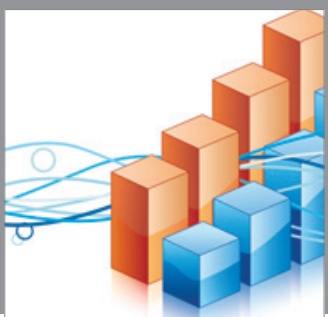

Advances in

Operations Research

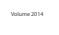

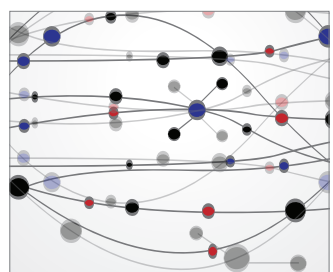

\section{The Scientific} World Journal
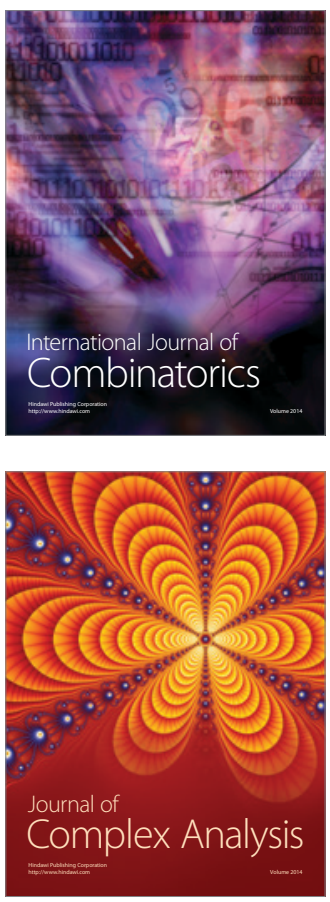

International Journal of

Mathematics and

Mathematical

Sciences
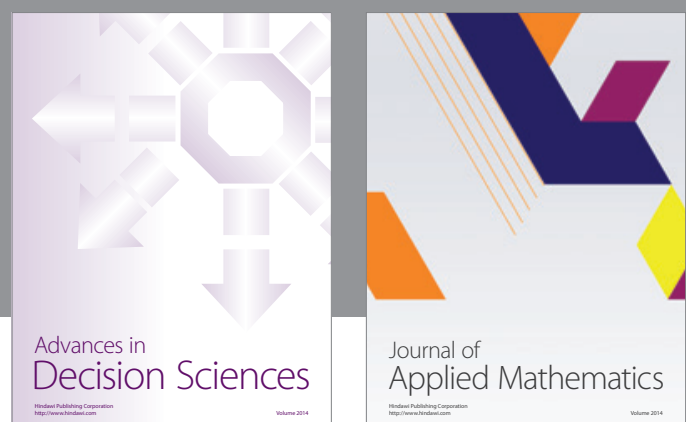

Journal of

Applied Mathematics
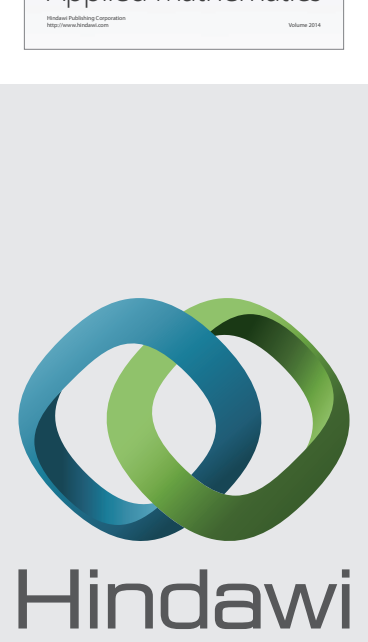

Submit your manuscripts at http://www.hindawi.com
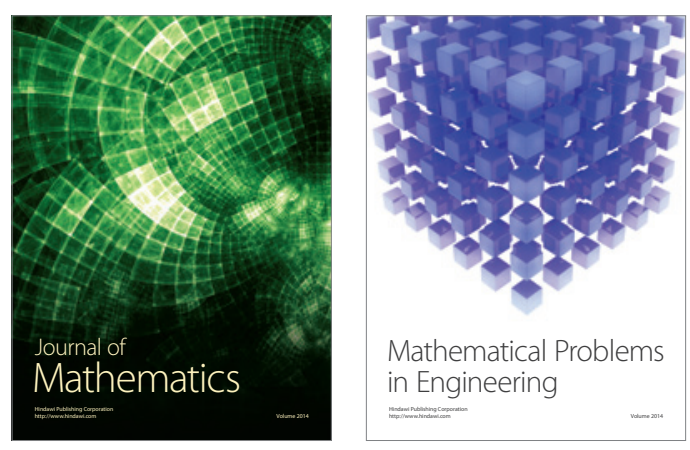

Mathematical Problems in Engineering
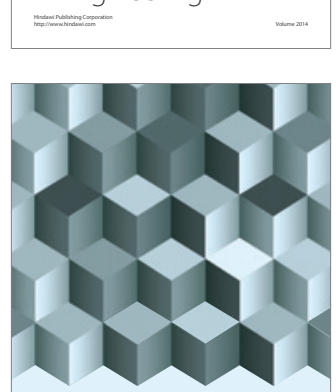

Journal of

Function Spaces
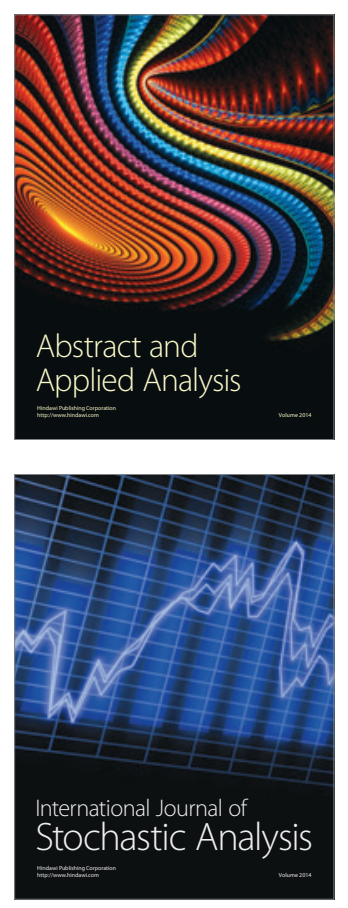

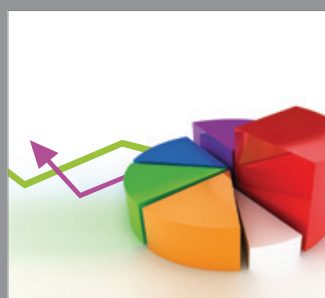

ournal of

Probability and Statistics

Promensencen
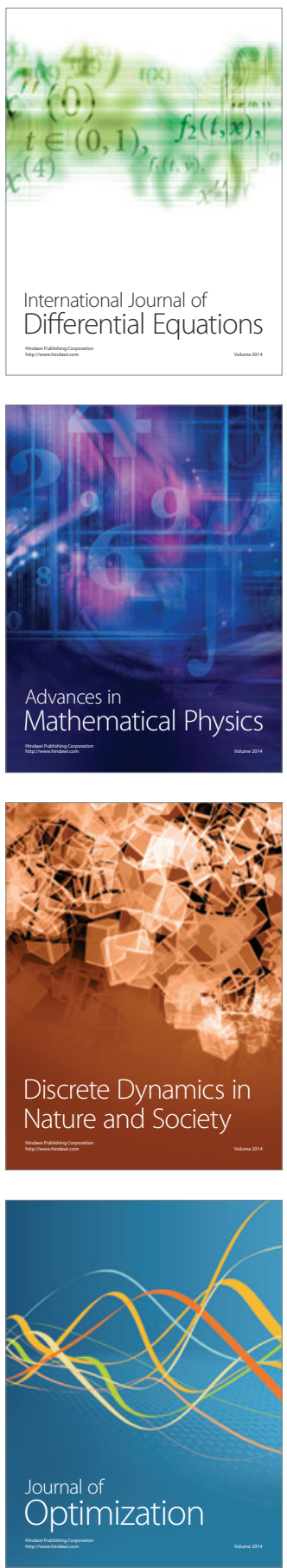